\title{
Computation of the free energy of solids
}

\author{
N. G. Almarza ${ }^{a}$ \\ Instituto de Química-Física Rocasolano, CSIC, Serrano 119, E-28006 Madrid, Spain
}

(Received 19 April 2007; accepted 9 May 2007; published online 6 June 2007)

\begin{abstract}
We present a procedure to compute the absolute free energy of solid phases by Monte Carlo simulation. The method is based on the so-called "Einstein-crystal" method of Frenkel and Ladd [J. Chem. Phys. 81, 3188 (1984)]. The new technique is more general and simplifies the calculation for systems with hard core interactions. In addition, the reference Einstein crystal is built up to fulfill translational invariance, which seems to reduce the system size dependence of the results. (C) 2007 American Institute of Physics. [DOI: 10.1063/1.2746231]
\end{abstract}

The free energy of crystalline solids can be computed using simulation following different techniques. ${ }^{1-4}$ At present the so-called "Einstein-crystal" proposed by Frenkel and $\operatorname{Ladd}^{3}$ is the usual procedure. This procedure is based on a thermodynamic integration on the canonical ensemble (fixed temperature $T$, volume $V$, and number of particles $N$ ) of the Helmholtz energy function along a reversible artificial way between the system of interest and an Einstein crystal (whose Helmholtz energy function can be computed analytically). The integration proposed in Ref. 3 can be sketched as

$$
\begin{aligned}
& U_{\lambda}(\mathbf{R} \mid \lambda)=(1-\lambda) U(\mathbf{R})+\lambda U_{E}(\mathbf{R}), \\
& F_{E}(N, V, T)=F(N, V, T)+\int_{0}^{1} \mathrm{~d} \lambda\left\langle\frac{\partial U_{\lambda}}{\partial \lambda}\right\rangle,
\end{aligned}
$$

where $U, U_{E}$, and $U_{\lambda}$ represent, respectively, the potential energies of the crystalline system, the Einstein crystal, and that of the system through the perturbation path, and $F_{E}$ and $F$ are the Helmholtz free energy of the Einstein crystal and that of the system. At $\lambda=0$ we have the crystalline system, whereas at $\lambda=1$ we get the so-called Einstein crystal (where the particles are attached to their perfect crystal positions through harmonic springs).

In their work, Polson et al. ${ }^{4}$ consider a crystal with fixed center of mass $(\mathrm{CM})$. We have found that it can be more convenient to allow the global Einstein crystal to translate.

We will consider for simplicity, a system of $N$ identical particles in a three dimensional space. Following similar procedures the equations for more complex systems can be found. The potential energy of the Einstein crystal of a system with $N$ identical particles can be written as

$$
U_{E}=\frac{1}{2} k_{E} \sum_{i=1}^{N}\left|\mathbf{r}_{i}-\mathbf{r}_{i}^{(0)}\right|^{2},
$$

where $k_{E}$ is the force constant, and $\mathbf{r}_{i}^{0}$ (the particle positions in the perfect crystal) are no longer fixed, but depend on the $\mathrm{CM}$ position. Let us denote as $\mathbf{b}_{i}$ the reference positions when the $\mathrm{CM}$ is located at the coordinates origin. It will be fulfilled,

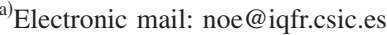

$$
\frac{1}{N} \sum_{i=1}^{N} \mathbf{b}_{i}=0
$$

and we will have for other position of the CM,

$$
\mathbf{r}_{i}^{(0)}=\mathbf{r}_{0}+\mathbf{b}_{i}=\left(\frac{1}{N} \sum_{i=1}^{N} \mathbf{r}_{i}\right)+\mathbf{b}_{i}
$$

where $\mathbf{r}_{0}$ is the CM position. We can write, then,

$$
U_{E}=\frac{k_{E}}{2} \sum_{i=1}^{N}\left|\mathbf{r}_{i}-\mathbf{b}_{i}+\frac{1}{N} \sum_{j=1}^{N}\left(\mathbf{r}_{j}-\mathbf{b}_{j}\right)\right|^{2} .
$$

Defining $\mathbf{w}_{i} \equiv \mathbf{r}_{i}-\mathbf{b}_{i}, U_{E}$ can be written as

$$
\begin{aligned}
& U_{E}=\frac{k_{E}}{2}\left[\sum_{i=1}^{N} \mathbf{w}_{i}^{2}-\frac{1}{N} \sum_{j=1}^{N} \sum_{k=1}^{N} \mathbf{w}_{j} \mathbf{w}_{k}\right], \\
& U_{E}=\frac{N k_{E}}{2}\left[\left\langle\mathbf{w}_{i}^{2}\right\rangle-\left\langle\mathbf{w}_{i}\right\rangle^{2}\right] .
\end{aligned}
$$

The total energy $H$ of the system can be written as

$$
H=\frac{m}{2} \sum_{i=1}^{N} \sum_{a} \dot{w}_{i a}^{2}+\frac{1}{2} \sum_{i=1}^{N} \sum_{j=1}^{N} \sum_{a} \sum_{b} w_{i a} w_{j b} U_{i a, j b},
$$

where $m$ is the mass of each particle, $a$ and $b$ refer to the three components $(x, y, z)$ of the coordinates $\mathbf{w}$ and velocities $\dot{\mathbf{w}}$, and the matrix elements $U_{i a, j b}$ are given by

$$
U_{i a, j b}=\delta_{a b} \cdot\left(\delta_{i j}-\frac{1}{N}\right) k_{E}
$$

where $\delta_{a b}$ is the Kronecker delta. In order to solve the statistical mechanics of the system we can compute the normal modes of the system by means of the diagonalization of the matrix given in Eq. (10). The eigenvalues are $\lambda=k_{E} / 2$ (with degeneracy $3 N-3$ ) and $\lambda=0$ (degeneracy equals to 3 ). The later corresponds to the global translation of the crystal. The partition function can now be written as ${ }^{5}$ 
TABLE I. Helmholtz free energy of fcc solids: $\beta \widetilde{F} \equiv=\beta F-3 N \log (\Lambda / \sigma)$. The number of particles is given by $N=4 l^{3}$.

\begin{tabular}{rlrcrr}
\hline \hline$l$ & \multicolumn{1}{c}{$\beta \tilde{F} / N$} & $l$ & $\beta \tilde{F} / N$ & $l$ & $\beta \tilde{F} / N$ \\
\hline 2 & $4.9299(8)$ & 3 & $4.96115(37)$ & 4 & $4.96307(30)$ \\
5 & $4.96219(22)$ & 6 & $4.96126(18)$ & 7 & $4.96068(21)$ \\
8 & $4.96039(20)$ & 9 & $4.95981(31)$ & 10 & $4.95979(21)$ \\
12 & $4.95933(14)$ & 15 & $4.95915(16)$ & & \\
\hline \hline
\end{tabular}

$$
Q_{E}=h^{-3 N} \frac{V}{N} z_{k}^{3 N} z_{u}^{3 N-3}
$$

where $h$ is the Planck constant, the term $V / N$ corresponds to the overall translational degrees of freedom (considering the particle indistinguishability), and $z_{k}$ and $z_{u}$ are given by

$$
\begin{aligned}
& z_{k}=\int_{-\infty}^{\infty} \mathrm{d} p e^{-\beta p^{2} / 2 m}=\left(\frac{2 \pi m}{\beta}\right)^{1 / 2}, \\
& z_{u}=\int_{-\infty}^{\infty} \mathrm{d} q e^{-\beta k_{E} q^{2} / 2}=\left(\frac{2 \pi}{\beta k_{E}}\right)^{1 / 2},
\end{aligned}
$$

where $\beta \equiv 1 / k_{B} T$, with $k_{B}$ being Boltzmann's constant. The free energy per particle, $f_{E}=-k_{B} T\left(\log Q_{E}\right) / N$, will be

$$
\begin{aligned}
\beta f_{E}(N, V, T)= & 3 \log \left(\frac{\Lambda}{\sigma}\right)+\frac{3}{2}\left(1-\frac{1}{N}\right) \log \frac{k_{E} \sigma^{2}}{2 \pi k_{B} T} \\
& +\frac{1}{N} \log \frac{N \sigma^{3}}{V}
\end{aligned}
$$

where $\Lambda \equiv h / z_{k}$ is the de Broglie thermal wavelength and we have introduced the parameter $\sigma$ as length unit. Notice that the result given in Eq. (14) is slightly different to that given by Polson et al. ${ }^{4}$ for the system subject to the CM constraint.

The procedure sketched in Eqs. (1) and (2) cannot be directly applied in systems with hard core interactions. Frenkel and Ladd $^{3}$ proposed a procedure to overcome such a difficulty. Here, we present a much simpler method that considers more general perturbation schemes than those given by Eq. (1) to define the path to reach the Einstein crystal limit. Consider a system potential energy given by

$$
U(\mathbf{R})=U_{h}(\mathbf{R})+U_{1}(\mathbf{R}),
$$

where $U_{h}(\mathbf{R})$ is the hard core interaction, defined by a sum of pair interactions,

$$
U_{h}(\mathbf{R})=\sum_{i<j} \nu_{\mathrm{HS}}\left(r_{i j}\right)
$$

where $\nu_{\mathrm{HS}}\left(r_{i j}\right)$ is the hard sphere potential. ${ }^{5}$ In order to present the procedure, we first invert the direction of the integration to write

$$
U_{\lambda}(\mathbf{R} \mid \lambda)=(1-\lambda) U_{E}(\mathbf{R})+\lambda U_{1}(\mathbf{R})+f_{h}(\lambda) U_{R}(\mathbf{R}),
$$

where $U_{R}$ is the sum of ramp potential interactions,

$$
U_{R}(\mathbf{R})=\sum_{i<j} \nu_{R}\left(r_{i j}\right),
$$

$$
\nu_{R}(r)=W_{0} \cdot(\sigma-r)
$$

for $r<\sigma$ and zero elsewhere, and $f_{h}(\lambda)$ was chosen to be

$$
f_{h}(\lambda)=\frac{\lambda}{1-\lambda} .
$$

Equations (19) and (20) introduce the hard core interaction in a progressive manner. The free energy of the system will be computed according to

$$
\beta F=\beta F_{E}+\beta \int_{0}^{1} \mathrm{~d} \lambda\left\langle\frac{\partial U_{\lambda}}{\partial \lambda}\right\rangle ;
$$

given the choice for $f_{h}$ given in Eq. (20), the integrand takes the form

$$
\frac{\partial U_{\lambda}}{\partial \lambda}=U_{1}(\mathbf{R})-U_{E}(\mathbf{R})+\frac{1}{(1-\lambda)^{2}} U_{R}(\mathbf{R}) .
$$

We have computed the Helmholtz energy for fcc crystals $^{3}$ of hard spheres at reduced density ${ }^{3,4} \rho \sigma^{3}$ $=1.04086$. The value of $k_{E}$ was chosen to keep approximately constant the oscillation of the particles from their equilibrium lattice positions $\left(k_{E} \sigma^{2}=180\right)$. We found that some care must be taken when choosing the parameter $W_{0}$ [see Eq. (19)]. Using $W_{0}^{*}=W_{0} \sigma / k_{B} T=1$ the fcc structure becomes unstable through the integration path and the system melts. No problems appear when taking $W_{0}^{*}=10$. We have carried out series of simulations for several system sizes given by $N=4 \times l^{3}$ (with $l$ being an integer number). In each case we performed the integration using $n$ values of $\lambda: \lambda_{i}$ $=(2 i-1) /(2 n), i=1,2,3, \ldots, n$. We used $n=50$. The integration given in Eq. (21) was computed as

$$
\beta F=\beta F_{E}+\frac{1}{n} \sum_{i=1}^{n}\left\langle\frac{U_{R}}{(1-\lambda)^{2}}-U_{E}\right\rangle_{\lambda},
$$

In Table I we present the results. The excess free energy can be computed as ${ }^{4}$

$$
\beta F_{\text {ex }} / N=\beta F / N-\left[\log \left(\rho \Lambda^{3}\right)-1+\frac{\log (2 \pi N)}{N}\right] .
$$

We can extrapolate the results to the thermodynamic limit, using

$$
\frac{\beta E_{\mathrm{ex}}(N)}{N}=\beta f_{\mathrm{ex}}+a_{1} \frac{1}{N} .
$$

Fitting our results to Eq. (25) (using the results for systems with $l \geqslant 5$, i.e., $\quad N \geqslant 500$ ), we found $\beta f_{\text {ex }}$ $=5.91891 \pm 0.00011$ and $a_{1}=-2.23 \pm 0.19$. The result for 
$\beta f_{\text {ex }}$ agrees with that from Ref. 4 (as expected), and for the hard sphere system here considered the size dependence of the results is much lower ${ }^{6}$ than the one obtained in Ref. 4.

We have presented an extension of the so-called Einstein crystal method of Frenkel and Ladd to compute the free energy of solid phases. The new method makes much more straightforward the calculation for systems with hard core interactions. In addition, the removal of the fixed CM constraint for the references system seems to improve the size dependence of the results.

The author acknowledges support from the Dirección General de Investigación Científica y Técnica un- der Grant No. FIS2004-02954-C03-01 and the Dirección General de Universidades e Investigación de la Comunidad de Madrid under Grant No. S0505/ESP/0299, program MOSSNOHO-CM.

${ }^{1}$ W. G. Hoover and F. H. Ree, J. Chem. Phys. 47, 4873 (1967).

${ }^{2}$ W. G. Hoover and F. H. Ree, J. Chem. Phys. 49, 3609 (1968).

${ }^{3}$ D. Frenkel and A. J. C. Ladd, J. Chem. Phys. 81, 3188 (1984).

${ }^{4}$ J. M. Polson, E. Trizac, S. Pronk, and D. Frenkel, J. Chem. Phys. 112, 5339 (2000).

${ }^{5}$ M. P. Allen and D. J. Tildesley, Computer Simulation of Liquids (Clarendon, Oxford, 1987).

${ }^{6}$ Our results did not seem to require the additive correction of $\log N / N$ to $\beta F_{e x} / N$ used in Ref. 4. 